\title{
A Novel Cyber Attack Detection Method in Networked Control Systems
}

\author{
Eman Mousavinejad ${ }^{\circledR}$, Student Member, IEEE, Fuwen Yang ${ }^{\circledR}$, Senior Member, IEEE, \\ Qing-Long Han ${ }^{(\mathbb{1}}$, Senior Member, IEEE, and Ljubo Vlacic ${ }^{(\mathbb{1})}$, Senior Member, IEEE
}

\begin{abstract}
This paper is concerned with cyber attack detection in a networked control system. A novel cyber attack detection method, which consists of two steps: 1) a prediction step and 2) a measurement update step, is developed. An estimation ellipsoid set is calculated through updating the prediction ellipsoid set with the current sensor measurement data. Based on the intersection between these two ellipsoid sets, two criteria are provided to detect cyber attacks injecting malicious signals into physical components (i.e., sensors and actuators) or into a communication network through which information among physical components is transmitted. There exists a cyber attack on sensors or a network exchanging data between sensors and controllers if there is no intersection between the prediction set and the estimation set updated at the current time instant. Actuators or network transmitting data between controllers and actuators are under a cyber attack if the prediction set has no intersection with the estimation set updated at the previous time instant. Recursive algorithms for the calculation of the two ellipsoid sets and for the attack detection on physical components and the communication network are proposed. Simulation results for two types of cyber attacks, namely a replay attack and a bias injection attack, are provided to demonstrate the effectiveness of the proposed method.
\end{abstract}

Index Terms-Bias injection attacks, cyber attack detection, networked control systems (NCSs), replay attacks, set-membership filtering.

\section{INTRODUCTION}

$\mathbf{N}$ OWADAYS, networked control systems (NCSs) have been playing a crucial role in many critical infrastructures including power grids, transportation systems, robotic platforms, target tracking, and system guidance and navigation [1]-[7]. Due to physical constraints or technological limitations, exchanged data among sensors, actuators and other networked components may be subject to maliciously

Manuscript received September 8, 2017; revised February 7, 2018 and May 14, 2018; accepted May 25, 2018. This work was supported by the Australian Research Council Discovery Project under Grant DP160103567. This paper was recommended by Associate Editor G.-P. Liu. (Corresponding author: Qing-Long Han.)

E. Mousavinejad, F. Yang, and L. Vlacic are with the School of Engineering, Griffith University, Gold Coast, QLD 4222, Australia (e-mail: eman.mousavinejad@griffithuni.edu.au; fuwen.yang@griffith.edu.au; 1.vlacic@griffith.edu.au).

Q.-L. Han is with the School of Software and Electrical Engineering, Swinburne University of Technology, Melbourne, VIC 3122, Australia (e-mail: qhan@swin.edu.au).

Color versions of one or more of the figures in this paper are available online at http://ieeexplore.ieee.org.

Digital Object Identifier 10.1109/TCYB.2018.2843358 destroy in common communication setting or wireless communication one [8]-[11]. This kind of phenomena is usually implemented by cyber-attackers with the aim of the enormous economy benefits or the disturbing social order. For real-world NCSs, representative examples of cyber attacks include an attack on Maroochy Shire Council's sewage control system in Queensland, Australia (2000) [12], Slammer worm on Davis-Besse power plant in Ohio, U.S. (2003) [13], and recent Stuxnet worm targeted many industrial control systems [14] (see [15] for more real-world cyber incidents). Obviously, NCSs are becoming more and more vulnerable to cyber attacks on both physical infrastructures and communication networks. As a result, security issues of NCSs need to be properly addressed to ensure that the systems are operating in a safe manner.

In securing NCSs, reliable attack detection is of utmost importance (see [16]-[20]). Generally speaking, when cyber attacks are detected and located in a timely fashion, the damage to overall systems can be controlled within a tolerable limit. For instance, bad data detectors in power systems or sensor networks are generally equipped to detect the deviation of the estimated states and provide an alarm operation [21]. Recently, considerable effort has been devoted to attack detection by means of different approaches, which can be arguably divided into three types: 1) Bayesian detection approaches [22], [23]; 2) weighted least square (WLS) approaches [24]-[27]; and 3) $\chi^{2}$-detector strategies based on Kalman filters [28]-[31]. In Bayesian detection, attack detection is usually formulated as a binary hypothesis test with prior probability. With the help of such a test, the performance limit of collaborative spectrum sensing is investigated in [22]. The closed form expression is proposed in [23] for an optimal attacking strategy of the Byzantines. For steady data, a WLS approach is widely employed to obtain the gain of various detectors, such as generalized likelihood ratio detectors [24], detectors based on the nuclear norm minimization and the low rank matrix factorization [25], and a heuristic approach for detecting abrupt changes in the system outputs in light of the singular value decomposition [27].

The next phase in enhancement of the NCSs' security is designing a resilient control system that provides NCSs with the ability to tolerate adversaries and recover from cyber attacks [32]. Investigating the problem of resilient control system design is beyond the scope of this paper, and therefore, the propagation of the output of the attack detection system throughout the control system is not considered in this paper. 
Instead, it has just been considered as an alarm signal for the time being.

When taking the dynamics of plants into account, a generally used state estimation method (i.e., Kalman filter) and attack detector (i.e., performance index test, also known as $\chi^{2}$-detector) have been extensively studied recently. For instance, in [28], a method to detect a replay attack on sensors of a control system is introduced. The proposed method requires the usage of an LQG controller and a Kalman filter with $\chi^{2}$-detector. In the method, the control signal is redesigned by adding a zero-mean authentication signal in order to increase the sensitivity of $\chi^{2}$-detector to a cyber attack. In [29], a Kalman filtering technique is utilized to estimate the state of the system based on the report from sensor measurement and the past state values. Also, an additional detector based on Euclidean distance along with $\chi^{2}$-detector is proposed to detect a false data injection attack on smart grid systems. A method in which KL divergence, a Kalman filter, and an LQG controller are adopted to design an optimal Neyman-Pearson detector for replay and covert attacks is proposed in [30]. In [31], a cosine similarity matching-based approach to design a detector is presented. The detector incorporates the Kalman filter estimation to measure any deviation between actual measurements and estimated values.

Up until now, most of attack detection approaches by means of the state estimation require systems noises in a stochastic framework, which provides a probabilistic state estimation. As is well-known, estimation with the nature of a probabilistic approach, particularly the Kalman filtering technique, requires the use of mean and variance to describe the state distributions modeled as random variables (usually white and Gaussian perturbations). However, in many realword applications, such as target tracking and attack, system guidance and navigation, $100 \%$ confidence in the state estimation is of paramount importance. Therefore, it is much more appropriate to model the state distributions in certain sets considering unknown-but-bounded (UBB) noises. In addition, a widely used attack detection scheme, i.e., the performance index test $\left(\chi^{2}\right.$-detector) relies on a robust residual signal to capture discrepancies between estimated behavior and that predicted by a model. In the Kalman filtering technique, the estimated and predicted states are single vectors and therefore, they cannot guarantee that state is included in some region. Besides, the resulting UBB noises are suboptimal for Kalman-type filtering, and thus could mostly reduce the reliability of attack detection. The requirement of setvalued estimation stimulates the development of ellipsoidal state estimation technique [33]. The idea of this technique is to provide a set of state estimates in state space which always contains the true state of the system [34], [35]. In other words, the actual estimate is a set in state space rather than a single vector. Consequently, this kind of technique is known as a set-membership or set-valued state estimation (filtering). Recently, the set-membership approach has been extensively studied in filtering problems (see [36]-[43]). On the other hand, an optimal ellipsoid with minimal size in set-membership estimation can be determined by using convex optimization approaches, which provides a pathway to improve the state estimation performance or detection performance.

Motivated by the discussion above and inspired by the Kalman and set-membership filtering technique, it is the objective of this paper to design a set-membership filter to detect cyber attacks in an NCS. We first propose a recursive convex optimization algorithm to compute the state estimate ellipsoid that guarantees to contain the true state for an attack-free system. The state estimate algorithm consists of a prediction step and a measurement update step. We then add two subalgorithms into our algorithm to detect cyber attacks by introducing two criteria based on the intersection between the prediction ellipsoid set and the estimation ellipsoid set updated with the measurement output. According to these two developed criteria combined with the ellipsoids calculated in prediction steps and measurement update steps, we determine the following.

1) The control signal is violated by a cyber attack if there is no intersection between the prediction ellipsoid set and the estimation ellipsoid set updated with the previous (one-step-behind) measurement output.

2) The sensor signal is targeted by a cyber attack if there is no intersection between the prediction ellipsoid set and the estimation ellipsoid set updated at the current time instant.

The main contribution of this paper is to design a novel cyber attack detection method which includes two steps: 1) a prediction step and 2) a measurement update step. In these two steps, our aim is to find an estimation set through updating the prediction set with the one yielding from the current measurement. In the measurement update step, the unconstrained state estimate is projected onto the linear measurement (output) constrained surface to obtain the updated estimation set by using Finsler's lemma. A cyber attack on sensors or a network exchanging data between sensors and controllers can be detected if there is no intersection between the estimation set updated at the current time instant and the prediction set, and a cyber attack on actuators or a network transmitting data between controllers and actuators can be detected if the prediction set has no intersection with the estimation set updated at the previous time instant. To the best of authors' knowledge, it is the very first time that not only has the setmembership filtering approach been utilized for the purpose of the attack detection problem but it has also been studied especially in distinguishing attacks on control signals from attacks on measurement outputs.

The rest of this paper is organized as follows. Section II presents the framework of the system of interest and the proposed attack detection strategy and formulates the setmembership filtering problem with measurement update for NCSs. Section III provides the design of an attack detection procedure based on the prediction and updated sets and then the associated algorithms are presented in this section. Section IV presents the mathematical model of the test system and also the two cyber attacks models used to demonstrate the effectiveness of the proposed method. The conclusion is drawn in Section V.

Notation: The notations used in this paper are fairly standard except where otherwise stated. The notation $X \succ 0$ means that 


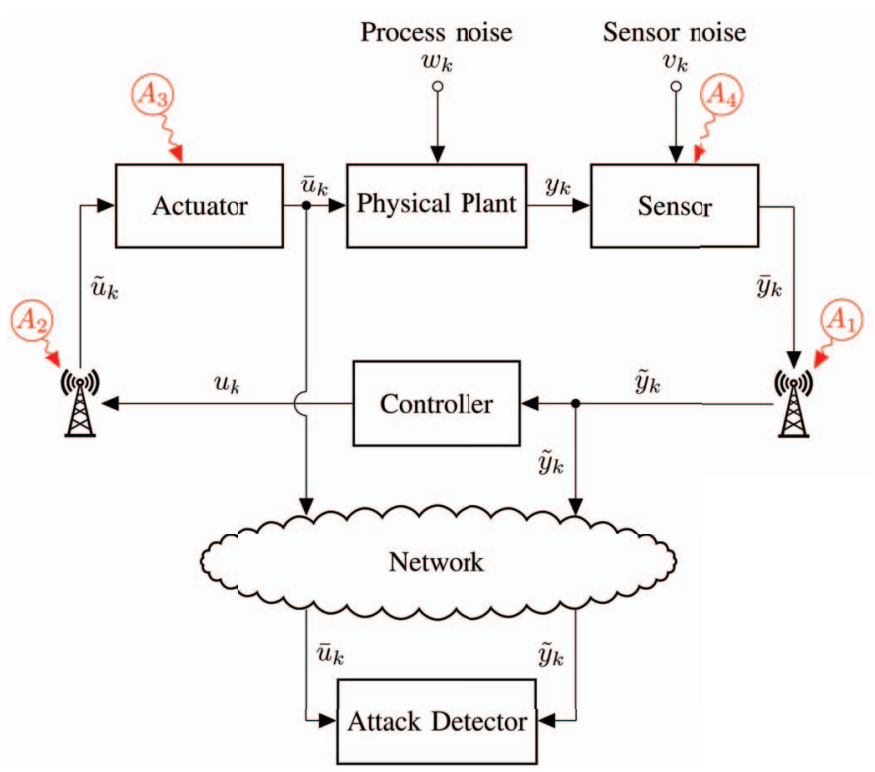

Fig. 1. Some possible attack points to an NCS.

$X$ is "positive definite." The superscript $\mathrm{T}$ stands for matrix transposition. The notation $\operatorname{Tr}(P)$ denotes the trace of $P$.

Ellipsoid: An ellipsoidal set is denoted as $\mathcal{X} \triangleq\{\zeta: \zeta=$ $c+E z,\|z\| \leq 1\}$, where $c \in \mathbb{R}^{n}$ is the center and $E \in \mathbb{R}^{n \times m}$ with $\operatorname{rank}(E)=m \leq n$ is the shape matrix of the ellipsoid. This representation is widely used to deal with all bounded ellipsoids including "flat" ellipsoids such as points or intervals. Let $E$ be a lower triangular matrix whose every diagonal element is positive. By a Cholesky factorization, one can conclude that $P=E E^{\mathrm{T}}>0$ and $z^{\mathrm{T}} z=(\zeta-c)^{\mathrm{T}} P^{-1}(\zeta-c) \leq 1$. Hence, an alternative representation of the ellipsoidal set is $\mathcal{X} \triangleq\left\{\zeta:(\zeta-c)^{\mathrm{T}} P^{-1}(\zeta-c) \leq 1\right\}$. The size of the ellipsoid is a function of the squares shape matrix $P$ which can be measured by means of $\operatorname{Tr}(P)$, i.e., the sum of squared semiaxes lengths [44].

\section{Problem Statement and Formulation}

Consider the framework of an NCS as shown in Fig. 1. In this framework, the controller receives the measurement data from the sensor and sends the control signal data to the actuator via a network which is often equipped with wireless communication [45], [46]. As the attack detector located at the remote site, the measurement data received from the wireless communication and the output of the actuator are sent to the attack detector via a communication network.

In practical situations, an attack on an NCS can be considered in two ways: 1) attacks on physical components, i.e., sensors and actuators and 2) attacks on the communication network exchanging data among the physical components [30]. For instance, as shown in Fig. 1:

1) an attack on a sensor can either be considered as an attack on the sensor itself, attack point $A_{4}$, by forcing it to transmit an incorrect signal, $\bar{y}_{k}$, or it can also be considered as an attack on the communication network between the sensor and the controller, attack point $A_{1}$, to force the communication network to transmit an incorrect signal, $\tilde{y}_{k}$;
2) an attack on an actuator can either be interpreted as an attack directly on the actuator, attack point $A_{3}$, to send an incorrect data, $\bar{u}_{k}$, to the plant or on the communication network, attack point $A_{2}$, to transmit an incorrect data, $\tilde{u}_{k}$, from the controller to the actuator.

In this paper, it will be considered that only the communication network exchanging data among the physical components is under cyber attacks (attacks points $A_{1}$ and $A_{2}$ ). Therefore, the measurement output and the control signal at the plant side can be considered as $\bar{y}_{k}=y_{k}$ and $\bar{u}_{k}=\tilde{u}_{k}$, respectively, and at the controller side can be denoted as $\tilde{y}_{k}$ and $u_{k}$, respectively. If there is no attack on this communication network, then $\tilde{u}_{k}=u_{k}$ and $\tilde{y}_{k}=y_{k}$. Also, it will be assumed that there is an ideal communication network sending data to the attack detector. Considering attacks on the communication network between the system's components and the proposed attack detection system is considerable part of the ongoing research but is currently beyond the scope of this paper. The assumption of an ideal communication network between the system's components and the proposed attack detector in Fig. 1 is due to just focusing on the detection of the attacks targeting the communication channels between the system's components, i.e., sensor, controller, and actuator.

In the following, a physical plant considered in the proposed framework, Fig. 1, is introduced in a mathematical way. The physical plant under consideration is a discrete time-varying system in the form of

$$
\begin{aligned}
x_{k+1} & =A_{k} x_{k}+F_{k} u_{k}+B_{k} w_{k} \\
y_{k} & =C_{k} x_{k}+D_{k} v_{k}
\end{aligned}
$$

where $x_{k} \in \mathbb{R}^{n}$ is the system state; $u_{k} \in \mathbb{R}^{l}$ is the known deterministic input; $y_{k} \in \mathbb{R}^{m}$ is the measurement output; $A_{k}, B_{k}, C_{k}$, $D_{k}$, and $F_{k}$ are known time-varying matrices with appropriate dimensions; and $w_{k} \in \mathbb{R}^{r}$ and $v_{k} \in \mathbb{R}^{p}$ are the process and measurement noises, respectively, and satisfy the following assumption.

Assumption 1: The process noise $w_{k}$ and measurement noise $v_{k}$ are $\mathrm{UBB}$, which are assumed to belong to the following specified ellipsoidal sets:

$$
\begin{gathered}
\mathcal{W}_{k} \triangleq\left\{w_{k}: w_{k}^{\mathrm{T}} Q_{k}^{-1} w_{k} \leq 1\right\} \\
\mathcal{V}_{k} \triangleq\left\{v_{k}: v_{k}^{\mathrm{T}} R_{k}^{-1} v_{k} \leq 1\right\}
\end{gathered}
$$

where $Q_{k}=Q_{k}^{\mathrm{T}} \succ 0$ and $R_{k}=R_{k}^{\mathrm{T}} \succ 0$ are known matrices with compatible dimensions.

Remark 1: There are some practical situations where noises on system inputs and measurement outputs are unknown but can be bounded. For instance, for a vehicle tracking system, the maximum acceleration of the vehicle is always known due to the engine dynamics and construction of the vehicle; however, it is not exactly known how much the acceleration is when the vehicle is running. Therefore, it is quite reasonable to assume that noises on the vehicle acceleration are bounded, and thus they can be considered as UBB process noises. Furthermore, bounded noises on measurement outputs can be viewed as some bound due to quantization errors and measurement errors. Therefore, an assumption of UBB noise 
does not require a prior knowledge of the actual pattern of the noise in the sense that only the knowledge of a bound on the realization is necessary.

Assumption 2: The initial state $x_{0}$ is assumed to belong to a given ellipsoid

$$
\mathcal{X}_{0 \mid 0} \triangleq\left\{x_{0}:\left(x_{0}-\hat{x}_{0 \mid 0}\right)^{\mathrm{T}} P_{0 \mid 0}^{-1}\left(x_{0}-\hat{x}_{0 \mid 0}\right) \leq 1\right\}
$$

where $\hat{x}_{0 \mid 0}$ is the given estimate of $x_{0}$, and $P_{0 \mid 0}=P_{0 \mid 0}^{\mathrm{T}} \succ 0$ is a known matrix.

Remark 2: In practical situations, due to some unknown errors and noises in measuring the initial state of the system, there might be some uncertainties in the measurement of the initial state. These uncertainties can be viewed as UBB noises as discussed in Remark 1. Therefore, Assumption 2 can be considered on the initial state in the deterministic framework.

In light of the set-membership filtering approach, our strategy to detect the cyber attack can be summarized as below.

1) The detection on cyber attacks for sensor measurement data.

a) If the updated ellipsoid set $\mathcal{X}_{k+1 \mid k+1}$ and the prediction ellipsoid set $\mathcal{X}_{k+1 \mid k}$ have the intersection, i.e., $\mathcal{X}_{k+1 \mid k+1} \bigcap \mathcal{X}_{k+1 \mid k} \neq \varnothing$, it can be concluded that there is no attack.

b) Otherwise, if the updated ellipsoid set $\mathcal{X}_{k+1 \mid k+1}$ has no intersection with the prediction ellipsoid set $\mathcal{X}_{k+1 \mid k}$, i.e., $\mathcal{X}_{k+1 \mid k+1} \bigcap \mathcal{X}_{k+1 \mid k}=\varnothing$, it indicates that the sensor measurement data is affected by the attack.

2) The detection on cyber attacks for control signal data.

a) If the prediction ellipsoid set $\mathcal{X}_{k+1 \mid k}$ and the previous updated ellipsoid set $\mathcal{X}_{k \mid k}$ have the intersection, i.e., $\mathcal{X}_{k \mid k} \bigcap \mathcal{X}_{k+1 \mid k} \neq \varnothing$, it indicates that there is no attack.

b) Otherwise, if the prediction ellipsoid set $\mathcal{X}_{k+1 \mid k}$ has no intersection with the previous updated ellipsoid set $\mathcal{X}_{k \mid k}$, i.e., $\mathcal{X}_{k \mid k} \cap \mathcal{X}_{k+1 \mid k}=\varnothing$, it indicates that the control signal data is affected by the attack.

Clearly, for the given ellipsoid $\mathcal{X}_{k \mid k}$ on previous state estimation, two key steps for attack detection are to determine the corresponding prediction ellipsoid $\mathcal{X}_{k+1 \mid k}$ (i.e., the prediction step) and the updated ellipsoid $\mathcal{X}_{k+1 \mid k+1}$ (i.e., the measurement update step) via the current sensor measurement data, respectively. In what follows, some preliminaries will be provided for these two steps.

\section{A. Prediction Step}

First, the prediction filter is considered in the form of

$$
\hat{x}_{k+1 \mid k}=G_{k} \hat{x}_{k \mid k}+F_{k} u_{k}
$$

where $\hat{x}_{k \mid k}$ is the estimation of the state $x_{k}$ and $G_{k}$ is the filter parameter to be determined.

For the given state estimation ellipsoid set $\mathcal{X}_{k \mid k}$ with the center $\hat{x}_{k \mid k}$ and the shape matrix $E_{k \mid k}$, the real state $x_{k}$ can be described by

$$
x_{k}=\hat{x}_{k \mid k}+E_{k \mid k} z
$$

Then, our goal is to obtain the prediction ellipsoid set

$$
\begin{aligned}
& \mathcal{X}_{k+1 \mid k} \triangleq\left\{x_{k+1}:\right. \\
&\left.\quad\left(x_{k+1}-\hat{x}_{k+1 \mid k}\right)^{\mathrm{T}} P_{k+1 \mid k}^{-1}\left(x_{k+1}-\hat{x}_{k+1 \mid k}\right) \leq 1\right\} .
\end{aligned}
$$

It should be pointed out that such an ellipsoid set contains the state $x_{k+1}$ for any value of the system noises belong to their specified sets.

\section{B. Measurement Update Step}

The update based on the current measurement is considered for the system (1) and (2), which is in the form of

$$
\hat{x}_{k+1 \mid k+1}=\hat{x}_{k+1 \mid k}+L_{k+1}\left(y_{k+1}-\hat{y}_{k+1 \mid k}\right)
$$

where $L_{k+1}$ is the filter parameter to be determined.

According to the prediction ellipsoid set $\mathcal{X}_{k+1 \mid k}$ given by (8), the state $x_{k+1}$ can be written as

$$
x_{k+1}=\hat{x}_{k+1 \mid k}+E_{k+1 \mid k} .
$$

Our objective is to update this prediction set with the one yielding from the current measurement $y_{k+1}$. In other words, we look for an updated ellipsoid set $\mathcal{X}_{k+1 \mid k+1}$ with the center $\hat{x}_{k+1 \mid k+1}$ and the shape matrix $E_{k+1 \mid k+1}$ for the state $x_{k+1}$, given by the current measurement information at the time instant $k+1$. Thus, the updated ellipsoid set should satisfy the condition

$$
\left(x_{k+1}-\hat{x}_{k+1 \mid k+1}\right)^{\mathrm{T}} P_{k+1 \mid k+1}^{-1}\left(x_{k+1}-\hat{x}_{k+1 \mid k+1}\right) \leq 1
$$

whenever the equality (output constraint)

$$
y_{k+1}=C_{k+1} \hat{x}_{k+1 \mid k}+C_{k+1} E_{k+1 \mid k} z+D_{k+1} v_{k+1}
$$

holds for some $\|z\| \leq 1$ and $v_{k+1} \in \mathcal{V}_{k+1}$.

\section{Attack Detection Using SET-MEMBERSHIP FILTERING}

In this section, a set-membership filter will be designed to solve the proposed cyber attack detection problem. First, in Section III-A, a prediction ellipsoid set is designed for state $x_{k+1}$. Then, Section III-B utilizes the method of projecting the unconstrained state estimate onto the linear output constrained surface in order to update the prediction ellipsoid set for state $x_{k+1}$ with the current measurement. Finally, two convex optimization problems and one algorithm are provided to expose the cyber attack diagnosis scheme.

\section{A. Design of the Prediction Ellipsoid Set}

From the system model (1), and the filter (6) and (7), the prediction error $x_{k+1}-\hat{x}_{k+1 \mid k}$ can be written as

$$
\begin{aligned}
x_{k+1}-\hat{x}_{k+1 \mid k} & =A_{k} x_{k}+F_{k} u_{k}+B_{k} w_{k}-G_{k} \hat{x}_{k \mid k}-F_{k} u_{k} \\
& =\left(A_{k}-G_{k}\right) \hat{x}_{k \mid k}+A_{k} E_{k \mid k} z+B_{k} w_{k} .
\end{aligned}
$$

Denoting

$$
\eta_{1, k}=\left[\begin{array}{c}
1 \\
z \\
w_{k}
\end{array}\right] .
$$


Equation (13) can be written in a compact form

$$
x_{k+1}-\hat{x}_{k+1 \mid k}=\Pi_{1}\left(\hat{x}_{k \mid k}\right) \eta_{1, k}
$$

where

$$
\Pi_{1}\left(\hat{x}_{k \mid k}\right)=\left[\begin{array}{lll}
\left(A_{k}-G_{k}\right) \hat{x}_{k \mid k} & A_{k} E_{k \mid k} & B_{k}
\end{array}\right] .
$$

Thus, the condition in (8)

$$
\left(x_{k+1}-\hat{x}_{k+1 \mid k}\right)^{\mathrm{T}} P_{k+1 \mid k}^{-1}\left(x_{k+1}-\hat{x}_{k+1 \mid k}\right) \leq 1
$$

can be written as

$$
\eta_{1, k}^{\mathrm{T}}\left[\Pi_{1}^{\mathrm{T}}\left(\hat{x}_{k \mid k}\right) P_{k+1 \mid k}^{-1} \Pi_{1}\left(\hat{x}_{k \mid k}\right)-\operatorname{diag}\{1,0,0\}\right] \eta_{1, k} \leq 0 .
$$

From (3) and (7), the unknown variables $w_{k}$ and $z$ satisfy the following constraints:

$$
\left\{\begin{array}{l}
w_{k}^{\mathrm{T}} Q_{k}^{-1} w_{k} \leq 1 \\
\|z\| \leq 1
\end{array}\right.
$$

which can be expressed in $\eta_{1, k}$ as

$$
\left\{\begin{array}{l}
\eta_{1, k}^{\mathrm{T}} \operatorname{diag}\left\{-1,0, Q_{k}^{-1}\right\} \eta_{1, k} \leq 0 \\
\eta_{1, k}^{\mathrm{T}} \operatorname{diag}\{-1, I, 0\} \eta_{1, k} \leq 0 .
\end{array}\right.
$$

Applying S-procedure [47] to (15) and (16), one has that the inequality (15) holds if there exist nonnegative scalars $\tau_{1, k}$ and $\tau_{2, k}$ such that

$$
\begin{aligned}
& \Pi_{1}^{\mathrm{T}}\left(\hat{x}_{k \mid k}\right) P_{k+1 \mid k}^{-1} \Pi_{1}\left(\hat{x}_{k \mid k}\right)-\operatorname{diag}\{1,0,0\} \\
& \quad-\tau_{1, k} \operatorname{diag}\{-1, I, 0\}-\tau_{2, k} \operatorname{diag}\left\{-1,0, Q_{k}^{-1}\right\} \leq 0 .
\end{aligned}
$$

In addition, inequality (17) can be rewritten in the following compact form:

$$
\begin{aligned}
& \Pi_{1}^{\mathrm{T}}\left(\hat{x}_{k \mid k}\right) P_{k+1 \mid k}^{-1} \Pi_{1}\left(\hat{x}_{k \mid k}\right) \\
& \quad-\operatorname{diag}\left\{1-\tau_{1, k}-\tau_{2, k}, \tau_{1, k} I, \tau_{2, k} Q_{k}^{-1}\right\} \leq 0 .
\end{aligned}
$$

Finally, denoting

$$
\Theta_{1, k}=-\operatorname{diag}\left\{1-\tau_{1, k}-\tau_{2, k}, \tau_{1, k} I, \tau_{2, k} Q_{k}^{-1}\right\}
$$

the above inequality can be further written as

$$
\Pi_{1}^{\mathrm{T}}\left(\hat{x}_{k \mid k}\right) P_{k+1 \mid k}^{-1} \Pi_{1}\left(\hat{x}_{k \mid k}\right)+\Theta_{1, k} \leq 0 .
$$

By using the Schur complements, (19) is equivalent to

$$
\left[\begin{array}{cc}
-P_{k+1 \mid k} & \Pi_{1}\left(\hat{x}_{k \mid k}\right) \\
\Pi_{1}^{\mathrm{T}}\left(\hat{x}_{k \mid k}\right) & \Theta_{1, k}
\end{array}\right] \leq 0 .
$$

Based on the discussion above, we have the following result.

Theorem 1: For the system (1) and (2), suppose that the state $x_{k}$ belongs to its state estimation ellipsoid $\left(x_{k}-\hat{x}_{k \mid k}\right)^{\mathrm{T}} P_{k \mid k}^{-1}\left(x_{k}-\hat{x}_{k \mid k}\right) \leq 1$. Then the one-step ahead state $x_{k+1}$ resides in its state prediction ellipsoid $\left(x_{k+1}-\hat{x}_{k+1 \mid k}\right)^{\mathrm{T}} P_{k+1 \mid k}^{-1}\left(x_{k+1}-\hat{x}_{k+1 \mid k}\right) \leq 1$, if there exist $P_{k+1 \mid k}>0, G_{k}, \tau_{1, k} \geq 0$, and $\tau_{2, k} \geq 0$ such that (20) holds. Moreover, the center of the state prediction ellipsoid is determined by (6).

Theorem 1 outlines the principle of determining the state prediction ellipsoid containing $x_{k+1}$. In order to determine an optimal ellipsoid and reduce the conservativeness, the convex optimization is performed in (21), in which the trace of $P_{k+1 \mid k}$ is optimized at each time step in an effort to find the prediction ellipsoid set with minimal size

$$
\begin{gathered}
\underset{P_{k+1 \mid k}>0, G_{k}, \tau_{1, k} \geq 0, \tau_{2, k} \geq 0}{\operatorname{minimize}} \\
\text { subject to }(20) .
\end{gathered}
$$

\section{B. Update on Prediction Ellipsoid Set With Current Measurement}

At this section, our purpose is to develop a scheme to determine the shape matrix $E_{k+1 \mid k+1}$ and the filter gain $L_{k+1}$ with the output constraint (12).

From the system (1) and (2), the prediction ellipsoid set (10), and the filter based on the current measurement (9), the current estimation error $x_{k+1}-\hat{x}_{k+1 \mid k+1}$ can be written as

$$
\begin{aligned}
x_{k+1}-\hat{x}_{k+1 \mid k+1}= & \hat{x}_{k+1 \mid k}+E_{k+1 \mid k} z-\hat{x}_{k+1 \mid k} \\
& -L_{k+1} C_{k+1} E_{k+1 \mid k} z-L_{k+1} D_{k+1} v_{k+1} \\
= & \left(I-L_{k+1} C_{k+1}\right) E_{k+1 \mid k} z-L_{k+1} D_{k+1} v_{k+1} .
\end{aligned}
$$

As the unknown variables are $z$ and $v_{k+1}$, we can define

$$
\eta_{2, k+1}=\left[\begin{array}{c}
1 \\
z \\
v_{k+1}
\end{array}\right] \text {. }
$$

Thus, the above estimation error dynamics can be written in a compact form

$$
x_{k+1}-\hat{x}_{k+1 \mid k+1}=\Pi_{2} \eta_{2, k+1}
$$

where

$$
\Pi_{2}=\left[\begin{array}{lll}
0 & \left(I-L_{k+1} C_{k+1}\right) E_{k+1 \mid k} & -L_{k+1} D_{k+1}
\end{array}\right] .
$$

Taking (23) into account, the condition (11) in Section II-B can be described as

$$
\eta_{2, k+1}^{\mathrm{T}}\left[\Pi_{2}^{\mathrm{T}} P_{k+1 \mid k+1}^{-1} \Pi_{2}-\operatorname{diag}\{1,0,0\}\right] \eta_{2, k+1} \leq 0 .
$$

On the other hand, from (4) and (10), the unknown variables $v_{k+1}$, and $z$ satisfy the following constraints:

$$
\left\{\begin{array}{l}
v_{k+1}^{\mathrm{T}} R_{k+1}^{-1} v_{k+1} \leq 1 \\
\|z\| \leq 1
\end{array}\right.
$$

which can be expressed in $\eta_{2, k+1}$ as

$$
\left\{\begin{array}{l}
\eta_{2, k+1}^{\mathrm{T}} \operatorname{diag}\left\{-1,0, R_{k+1}^{-1}\right\} \eta_{2, k+1} \leq 0 \\
\eta_{2, k+1}^{\mathrm{T}} \operatorname{diag}\{-1, I, 0\} \eta_{2, k+1} \leq 0 .
\end{array}\right.
$$

By applying S-procedure to (24) and (25), one has that the inequality (24) is true if there exist nonnegative scalars $\tau_{3, k}$ and $\tau_{4, k}$ such that

$$
\begin{aligned}
& \Pi_{2}^{\mathrm{T}} P_{k+1 \mid k+1}^{-1} \Pi_{2}-\operatorname{diag}\{1,0,0\} \\
& \quad-\tau_{3, k} \operatorname{diag}\{-1, I, 0\}-\tau_{4, k} \operatorname{diag}\left\{-1,0, R_{k+1}^{-1}\right\} \leq 0
\end{aligned}
$$

which can be written in the following compact form:

$\Pi_{2}^{\mathrm{T}} P_{k+1 \mid k+1}^{-1} \Pi_{2}-\operatorname{diag}\left\{1-\tau_{3, k}-\tau_{4, k}, \tau_{3, k} I, \tau_{4, k} R_{k+1}^{-1}\right\} \leq 0$.

Denote

$$
\Theta_{2, k}=\operatorname{diag}\left\{1-\tau_{3, k}-\tau_{4, k}, \tau_{3, k} I, \tau_{4, k} R_{k+1}^{-1}\right\} .
$$

It follows from (27) that:

$$
\Pi_{2}^{\mathrm{T}} P_{k+1 \mid k+1}^{-1} \Pi_{2}-\Theta_{2, k} \leq 0 .
$$


Now, we deal with the output constraint (12) in Section II-B. First, it can be described by

$$
\Pi_{y}\left(\hat{x}_{k+1 \mid k}\right) \eta_{2, k+1}=0
$$

where

$\Pi_{y}\left(\hat{x}_{k+1 \mid k}\right)=\left[\begin{array}{lll}C_{k+1} \hat{x}_{k+1 \mid k}-y_{k+1} & C_{k+1} E_{k+1 \mid k} & D_{k+1}\end{array}\right]$.

By virtue of Finsler's lemma [47], the inequality (24) [i.e., (11)] under constraint (30) [i.e., (12)] is true if there exists an $N_{k+1}$ such that

$$
\begin{aligned}
& \Pi_{2}^{\mathrm{T}} P_{k+1 \mid k+1}^{-1} \Pi_{2}-\Theta_{2, k} \\
& \quad+N_{k+1}^{\mathrm{T}} \Pi_{y}\left(\hat{x}_{k+1 \mid k}\right)+\Pi_{y}^{\mathrm{T}}\left(\hat{x}_{k+1 \mid k}\right) N_{k+1} \leq 0 .
\end{aligned}
$$

For the purpose of simplicity, denote

$$
\Theta_{3, k}=-\Theta_{2, k}+N_{k+1}^{\mathrm{T}} \Pi_{y}\left(\hat{x}_{k+1 \mid k}\right)+\Pi_{y}^{\mathrm{T}}\left(\hat{x}_{k+1 \mid k}\right) N_{k+1} .
$$

Then, by using Schur complements, (32) is equivalent to

$$
\left[\begin{array}{cc}
-P_{k+1 \mid k+1} & \Pi_{2} \\
\Pi_{2}^{\mathrm{T}} & \Theta_{3, k}
\end{array}\right] \leq 0 .
$$

Then we arrive at the following theorem.

Theorem 2: For the system (1) and (2), if the state $x_{k+1}$ belongs to its state prediction ellipsoid

$$
\left(x_{k+1}-\hat{x}_{k+1 \mid k}\right)^{\mathrm{T}} P_{k+1 \mid k}^{-1}\left(x_{k+1}-\hat{x}_{k+1 \mid k}\right) \leq 1
$$

then such a state also resides in its updated state estimation ellipsoid

$$
\left(x_{k+1}-\hat{x}_{k+1 \mid k+1}\right)^{\mathrm{T}} P_{k+1 \mid k+1}^{-1}\left(x_{k+1}-\hat{x}_{k+1 \mid k+1}\right) \leq 1
$$

with the center determined by (9), where $P_{k+1 \mid k+1}>0$ satisfies matrix inequality (34) with other decision variables $L_{k+1}$, $\tau_{3, k} \geq 0, \tau_{4, k} \geq 0$, and $N_{k+1}$.

Now the convex optimization approach is applied to determine an optimal ellipsoid with the minimal size. $P_{k+1 \mid k+1}$ is obtained by solving the following optimization problem:

$$
\begin{aligned}
& \underset{P_{k+1 \mid k+1}>0, L_{k+1}, N_{k+1}, \tau_{3 k} \geq 0, \tau_{4 k} \geq 0}{\operatorname{minimize}} \operatorname{Tr}\left(P_{k+1 \mid k+1}\right) \\
& \text { subject to (34). }
\end{aligned}
$$

Remark 3: From Theorems 1 and 2, the optimization problems (21) and (35) are based on recursive linear matrix inequalities (LMIs) (20) and (34) which are linear to $P_{k+1 \mid k}$, $P_{k+1 \mid k+1}, G_{k}, L_{k+1}, N_{k+1}$, and $\tau_{m, k}, m=1,2, \ldots, 4$. Hence, these optimization problems can be solved by some existing semidefinite programming via an interior-point algorithm at each time step. The interior-point algorithm usually has a polynomial-time complexity $\mathcal{O}\left(\ell \mathcal{M}^{3}\right)$, where $\ell$ is the total row size of the main LMIs and $\mathcal{M}$ is the total number of scalar decision variables of the main LMIs (20) and (34). Since $\ell$ and $\mathcal{M}$ are dependent on $n_{x}, n_{u}, n_{y}, n_{w}$, and $n_{v}$, where $n$ denotes the number of its subscript, the computational complexity of the developed recursive algorithm depends polynomially on the dimensions of systems parameter variables [39]. As discussed in [48], in practical situations, interior-point methods for semidefinite programs are competitive with other methods for small programs and substantially faster for medium and large-scale problems.

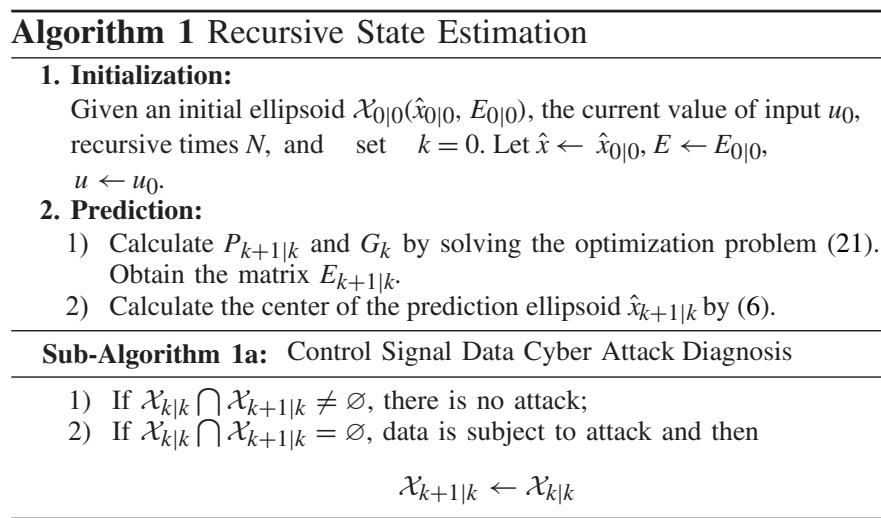

3. Measurement Update:

1) Calculate $P_{k+1 \mid k+1}$ and $L_{k+1}$ by solving the optimization problem (35). Obtain the new $E_{k+1 \mid k+1}$.

2) Calculate the center of the updated estimation ellipsoid $\hat{x}_{k+1 \mid k+1}$ by (9). Sensor Measurement Data Cyber Attack

Sub-Algorithm 1b: $\begin{aligned} & \text { Sensor M } \\ & \text { Diagnosis }\end{aligned}$

1) If $\mathcal{X}_{k+1 \mid k+1} \cap \mathcal{X}_{k+1 \mid k} \neq \varnothing$, there is no attack;

2) If $\mathcal{X}_{k+1 \mid k+1} \cap \mathcal{X}_{k+1 \mid k}=\varnothing$, data is subject to attack and then

$$
\mathcal{X}_{k+1 \mid k+1} \leftarrow \mathcal{X}_{k+1 \mid k}
$$

4. Loop

If $k==N$ then Exit, Else $k \leftarrow k+1$ and Goto Prediction step.

\section{Recursive Algorithm for Attack Diagnosis}

The recursive algorithm based on the set-membership filtering to compute the state ellipsoids so that a cyber attack can be detected is summarized below.

Algorithm 1 recursively computes the prediction ellipsoid $\mathcal{X}_{k+1 \mid k}$ and its update $\mathcal{X}_{k+1 \mid k+1}$ with the current measurement $y_{k+1}$. The two subalgorithms are proposed to detect cyber attacks that affect control signals and sensor measurements.

Remark 4: Theorems 1 and 2 show that the proposed ellipsoidal set-membership filtering problem can be converted into the feasibility problem of a set of recursive LMIs (20) and (34) to determine two optimal ellipsoidal sets. Ideally, in an attackfree system, the two sets will always have intersection since they both contain the true state $x_{k+1}$. However, if there is an attack on the system, one of the two sets does not contain the true state since the center of that set is affected by the attack. Therefore, one can conclude that there must be no intersection between the ellipsoidal sets as a results of the attacks impact on their centers. However, this effect may result in infeasibility of Theorems 1 and 2. As one can see in subalgorithms $1 \mathrm{a}$ and $1 \mathrm{~b}$, in order to overcome this situation, if there is an attack compromising the control signal then $\mathcal{X}_{k+1 \mid k} \leftarrow \mathcal{X}_{k \mid k}$, and if there is an attack targeting the measurement outputs then $\mathcal{X}_{k+1 \mid k+1} \leftarrow \mathcal{X}_{k+1 \mid k}$. These modifications in the ellipsoidal sets recover them so that they become free of the attack for their succeeding steps in Algorithm 1. Consequently, the feasibility of the proposed LMI problems can be kept at each time step.

Remark 5: From subalgorithm 1a, if there is an attack that violates the control signal commencing at time $k$, the input of the system $u_{k}$ is affected by the attack. Therefore, the prediction ellipsoid set $\mathcal{X}_{k+1 \mid k}$ is affected by the attack. However, the updated estimation ellipsoid $\mathcal{X}_{k \mid k}$, which is based 
on the prediction ellipsoid set at time $k-1$, i.e., $\mathcal{X}_{k \mid k-1}$, is not affected by the attack. Thus, one can conclude that if there is no intersection between the prediction ellipsoid set $\mathcal{X}_{k+1 \mid k}$ and the updated estimation ellipsoid set $\mathcal{X}_{k \mid k}$, the control signal is compromised by an attack.

Remark 6: From subalgorithm 1b, if the sensor measurement is violated by an attack at time $k+1$, the output of the sensor $y_{k+1}$ is affected by the attack. In this case, the estimation ellipsoid set $\mathcal{X}_{k+1 \mid k+1}$ updated with the current measurement $y_{k+1}$ is affected by the attack. However, the prediction ellipsoid set $\mathcal{X}_{k+1 \mid k}$, which is based on the measurement at time $k$, i.e., $y_{k}$, is not affected by the attack. Thus, it can be indicated that if there is no intersection between the prediction ellipsoid set and the updated estimation ellipsoid set, the sensor measurement is compromised by an attack.

Remark 7: As the existence of noises and parameter uncertainties in many practical situations is unavoidable, the proposed method is aimed at considering the noises as an inherent property of the system so that only abrupt changes deliberately brought into the system by an attacker can be detected in a timely fashion. Considering noises directly into solving the two LMIs in (20) and (34) can improve the performance of the proposed detection algorithm through focusing just on attacks. Therefore, the detection algorithm can tolerate the changes caused by the system noises and therefore, it does not recognize them as an attack. In other words, the proposed attack detection system is able to distinguish the system's changes due to process and measurement noises from the attacker's deliberate changes into the system, and as a result, the proposed attack detection method can tolerate the system changes due to noises and just report the abrupt changes brought to the system by the attacker.

Remark 8: It should be pointed out that if the abrupt change caused by an attacker is fairly small at its time of occurrence, the proposed detection algorithm cannot recognize it, i.e., there might be an intersection between the two ellipsoidal sets. This situation continues till the change into the control signals and/or measurement outputs can reach the certain level (threshold) where there is no intersection between the two ellipsoid sets. As is well-known, most of attack detection approaches require a threshold and if the abrupt changes are below of this threshold, they cannot be detected. However, it is noteworthy that the size of these ellipsoid sets are minimized through optimization problems (21) and (35), which implies that the threshold for the proposed attack detection algorithm reaches its minimum value by minimizing the size of these sets. Consequently, the optimization approach in our proposed attack detection algorithm is aimed at minimizing the attacks damages which may be tolerated by a resilient control algorithm.

\section{Case Studies}

\section{A. Test System}

In this section, a vehicle tracking system is considered as the physical plant including a global positioning system (GPS) receiver. The schematic of a vehicle tracking system is illustrated in Fig. 2. The main objective of a tracking system is to

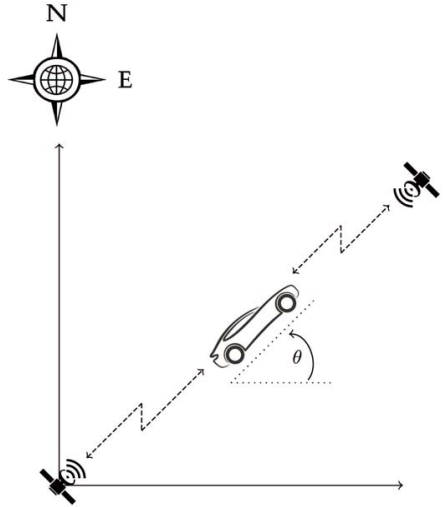

Fig. 2. Vehicle tracking system with GPS.

estimate the state trajectories of a vehicle. As a result, accuracy in determination of a vehicle position with respect to a fixed reference frame (absolute positioning) is of practical importance to navigate autonomous vehicles. If the navigation system's components, i.e., sensors, actuators, and controllers are connected through a wireless communication system as shown in Fig. 1, this system becomes more vulnerable to cyber attacks. Consequently, it is our objective to evaluate the performance of the proposed attack detection method for a vehicle tracking system.

The vehicle dynamics model with a known commanded acceleration is

$$
\dot{x}(t)=\left[\begin{array}{llll}
0 & 0 & 1 & 0 \\
0 & 0 & 0 & 1 \\
0 & 0 & 0 & 0 \\
0 & 0 & 0 & 0
\end{array}\right] x(t)+\left[\begin{array}{c}
0 \\
0 \\
\sin \theta \\
\cos \theta
\end{array}\right] u(t)
$$

where the first two components of the state vector $x(t)$ are latitude and longitude positions, and the last two components are latitude and longitude velocities, respectively. $u(t)$ is the commanded acceleration and $\theta$ is the road orientation angle measured counterclockwise from due east.

Considering finite difference approximation of derivatives as

$$
\dot{x}(t) \approx \frac{x_{t+1}-x_{t}}{T}
$$

through discretization process with the sample time $T$ and changing the subscript from $t$ to $k \in \mathbb{Z}^{+}$, the discrete-time vehicle dynamics model is described by [49]

$$
x_{k+1}=\left[\begin{array}{llll}
1 & 0 & T & 0 \\
0 & 1 & 0 & T \\
0 & 0 & 1 & 0 \\
0 & 0 & 0 & 1
\end{array}\right] x_{k}+\left[\begin{array}{c}
0 \\
0 \\
T \sin \theta \\
T \cos \theta
\end{array}\right] u_{k}+\left[\begin{array}{l}
2 \\
1 \\
1 \\
1
\end{array}\right] w_{k}
$$

where $w_{k}$ represents process noises due to potholes which belongs to a specified ellipsoidal set.

The GPS measurement equation can be written as

$$
y_{k}=\left[\begin{array}{llll}
1 & 0 & 0 & 0 \\
0 & 1 & 0 & 0
\end{array}\right] x_{k}+\left[\begin{array}{l}
1 \\
2
\end{array}\right] v_{k}
$$

where $y_{k}$ is the GPS measurement and $v_{k}$ is the GPS measurement noise. 
$T$ is chosen as $1.1 \mathrm{~s}$. The road orientation angle is chosen as a constant, $\theta=60^{\circ}$. The control signal is considered as a known commanded acceleration $u_{k}$ with a value which is set to $\pm 0.1 \mathrm{~m} / \mathrm{s}^{2}$, as if the vehicle alternately accelerating and decelerating in traffic. $w_{k}$ and $v_{k}$ are assumed as $0.5 \sin (2 k)$ and $0.5 \sin (30 k)$, respectively. $Q_{k}=1$ and $R_{k}=1$. The initial state is set as $x_{0}=\left[\begin{array}{llll}5 & 5 \sqrt{3} & 2 & 2 \sqrt{3}\end{array}\right]^{\mathrm{T}}$ which belongs to the ellipsoid

$$
\mathcal{X}_{0 \mid 0} \triangleq\left\{x_{0}:\left(x_{0}-\hat{x}_{0 \mid 0}\right)^{\mathrm{T}} P_{0 \mid 0}^{-1}\left(x_{0}-\hat{x}_{0 \mid 0}\right) \leq 1\right\} .
$$

$\hat{x}_{0 \mid 0}$ and $P_{0 \mid 0}$ are set to $\hat{x}_{0 \mid 0}=\left[\begin{array}{llll}0 & 7 & 0 & 3\end{array}\right]^{\mathrm{T}}$, and $P_{0 \mid 0}=$ $\operatorname{diag}\{100,100,10,10\}$.

\section{B. Cyber Attack Model}

In the following sections, we consider two types of the deception attacks, namely replay attacks and bias injection attacks, that affect the sensor measurement data and control signal data transmitted via a communication network, respectively.

In this paper, there are some assumptions about the ability of an attacker to perform a successful attack. Considering these assumptions, the attacker is able to modify the true values of $y_{k}$ and $u_{k}$ to arbitrary ones. The required assumptions will be explained in the following sections.

1) Replay Attacks on Sensor Measurement Data: A successful replay attack does not need a priori knowledge of the system components. As discussed in [50], a successful replay attack can be separated into two phases. In the first phase, which is known as disclosure attacks, an attacker must have this ability to gather sequences of data from the sensors measurements through violating the disclosure resources, i.e., set of accessible sensor channels. Having this ability, the attacker starts to record sequences of data from sensors communication channels without inserting any input to the system. Then, in the second phase the attacker attacks the system by replaying the recorded data to the system via tampering those channels from which data have been previously recorded.

It is assumed that the attacker can record sensor's measurement data from $k_{i}$ till $k_{r}$ with the window size $\tau=k_{r}-k_{i}$ in the first phase. Then, in the second phase, the attacker replays the recorded data to the system from $k=k_{r}+d$ till the end on the attack at $k=k_{f}$, where $d$ is the delay between the recording time and replaying time. This attack can be modeled as

$$
a_{k}^{y}=y_{k-\tau}-y_{k} .
$$

Thus, the sensor's data affected by the attack is

$$
\tilde{y}_{k}=y_{k}+a_{k}^{y} \text {. }
$$

2) Bias Injection Attacks on Control Signal Data: In a bias injection attack, the attacker injects a constant bias into the system [50]. A successful bias injection attack requires the knowledge of the system model. Although the attacker must be able to compromise the integrity of control signal data, there is no need for the attacker to have a prior knowledge of those channels exchanging control signal data. The bias injection attack on the control signal can be modeled as

$$
a_{k}^{u}=\delta_{u}
$$

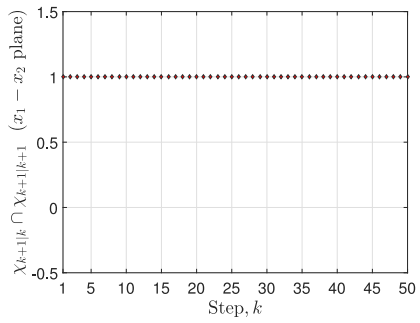

(a)

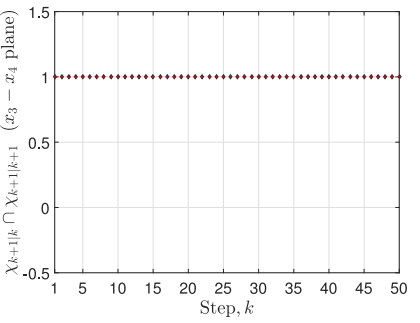

(b)
Fig. 3. Intersection between the prediction ellipsoid set $\chi_{k+1 \mid k}$ and the updated estimation ellipsoid set $\chi_{k+1 \mid k+1}$ at (a) $x_{1}-x_{2}$ phase-plane and (b) $x_{3}-x_{4}$ phase-plane (" 1 " indicates there exists intersection and " 0 " indicates there is no intersection).

where $\delta_{u}$ is a constant value injected by the attacker. Therefore, the control signal's data affected by the attack is

$$
\tilde{u}_{k}=u_{k}+a_{k}^{u} \text {. }
$$

To illustrate the effectiveness of the proposed method, the following three cases are considered. The simulation results are obtained under MATLAB 8.6 with YALMIP and the solver Sdpt 3 during 50 sampling steps.

\section{Attack-Free System}

Consider that the system (37) and (38) is an attack-free system, i.e., $\tilde{u}_{k}=u_{k}$ and $\tilde{y}_{k}=y_{k}$. Since in the attack-free system the prediction ellipsoid set and the updated estimation ellipsoid set both contain the true state, there must always exist the intersection between these two ellipsoid sets. Fig. 3 confirms the existence of the intersection between the two sets which are both projected onto the first two components of the state $\left(x_{1}-x_{2}\right.$ phase-plane) and the last two components of the state $\left(x_{3}-x_{4}\right.$ phase-plane) shown in Fig. 3(a) and (b), respectively.

\section{Replay Attack on Sensor Data}

In this case, it is considered that the attacker implements a replay attack on the sensor measurement data via targeting the wireless communication channel between the sensor and the controller, i.e., the attack point $A_{1}$ shown in Fig. 1. In the simulation, it is assumed that the attacker records the sensor's data from step $k=14$ till $k=19$ and then replaces the sensor's data with the recorded data from step $k=30$ to $k=35$. From (38), it can be observed that the sensors measure the data of the first two components of the state, i.e., $x_{1}$ and $x_{2}$. Therefore, the attack can be detected by projecting the prediction and updated ellipsoid sets onto the $x_{1}-x_{2}$ phase-plane.

Fig. 4 demonstrates the sequence of the intersection between the prediction and the updated estimation ellipsoid sets during the simulation time. In particular, as the replay attack starts at $k=30$, the prediction ellipsoid set is calculated from the sensor measurement data obtained at $k=29$ when there is no attack. However, the estimation ellipsoid set is updated with the current sensor measurement data at $k=30$. Therefore, it is expected from the subalgorithm $1 \mathrm{~b}$ that there must be no 


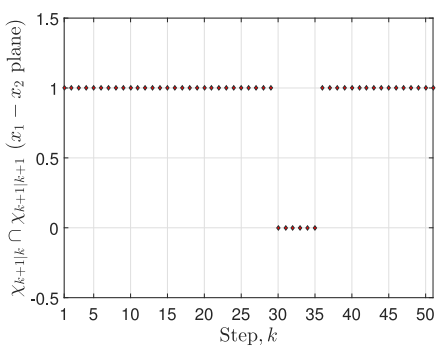

Fig. 4. Intersection between the prediction ellipsoid set $\chi_{k+1 \mid k}$ and the updated estimation ellipsoid set $\chi_{k+1 \mid k+1}$ at $x_{1}-x_{2}$ phase-plane ( 1 indicates there exists intersection and 0 indicates there is no intersection).

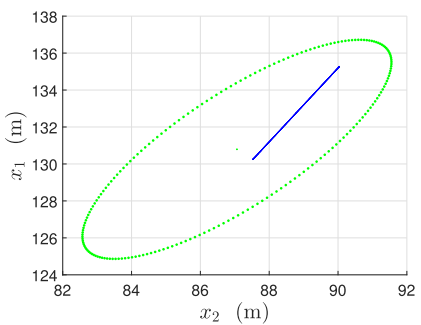

(a)

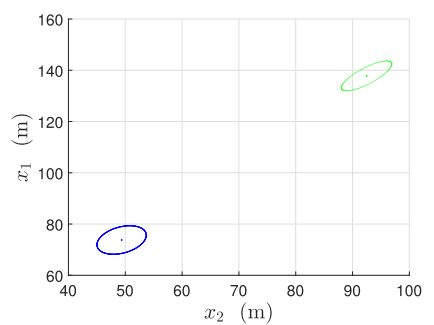

(b)

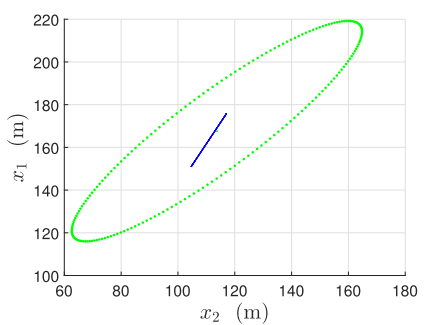

(c)

Fig. 5. Prediction ellipsoid $\chi_{k+1 \mid k}$ (green, dotted line) and the updated estimation ellipsoid $\chi_{k+1 \mid k+1}$ (blue, solid line). (a) Step 29. (b) Step 30. (c) Step 36 (the updated estimation ellipsoid at steps 30 and 36 are magnified with the ratio of $10^{7}$ and 10 , respectively).

intersection between the prediction ellipsoid set and the estimation ellipsoid set updated at the current time instant when the attack starts at $k=30$, i.e., $\chi_{30 \mid 29} \cap \chi_{30 \mid 30}=\varnothing$, as depicted in Fig. 5(b).

From Remark 4, since $\mathcal{X}_{k+1 \mid k+1} \leftarrow \mathcal{X}_{k+1 \mid k}$ from $k=30$ to $k=35$, one can conclude that the prediction ellipsoid set remains free of the attack when the system is under the replay attack. Therefore, once the attack finishes at $k=36$, the prediction ellipsoid set and the estimation ellipsoid set updated with the current sensor measurement data at $k=36$ are both free of attack. As a result, from the subalgorithm $1 \mathrm{~b}$, there must be intersection between these two sets, i.e., $\chi_{36 \mid 35} \cap \chi_{36 \mid 36} \neq \varnothing$, as shown in Fig. 5(c).

At $k=29$ the system is free of the attack, so from the subalgorithm $1 \mathrm{~b}$ it can be concluded that there exists intersection between the two sets as illustrated in Fig. 5(a).

\section{E. Bias Injection Attack on Control Signal Data}

In this case, it is considered that the attacker carries out a bias injection attack on the control signal via targeting the wireless communication channel between the actuator and the

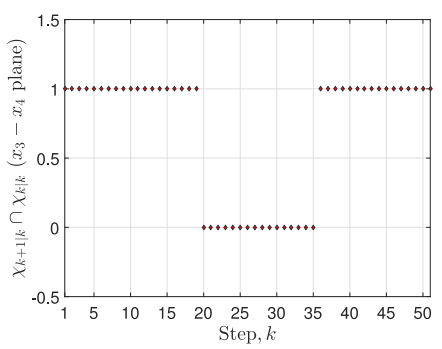

Fig. 6. Intersection between the prediction ellipsoid set $\chi_{k+1 \mid k}$ and the previous updated estimation ellipsoid set $\chi_{k \mid k}$ at $x_{3}-x_{4}$ phase-plane ( 1 indicates there exists intersection and 0 indicates there is no intersection).

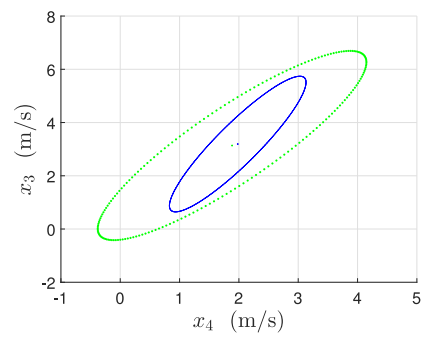

(a)

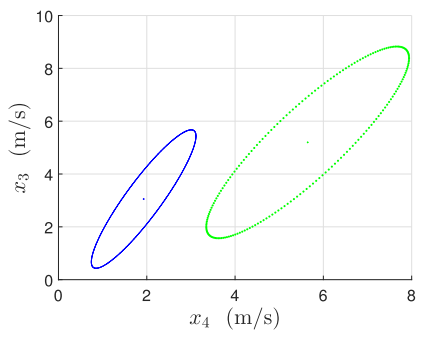

(b)

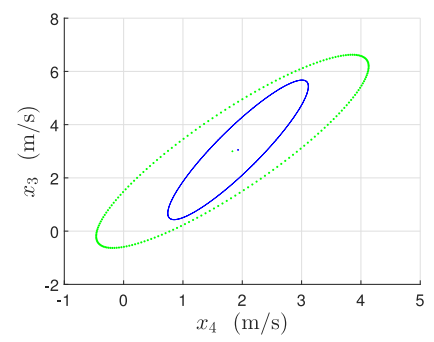

(c)

Fig. 7. Prediction ellipsoid $\chi_{k+1 \mid k}$ (green, dotted line) and the updated estimation ellipsoid $\chi_{k \mid k}$ (blue, solid line). (a) Step 19. (b) Step 20. (c) Step 36.

controller, i.e., the attack point $A_{2}$ shown in Fig. 1. In the simulation, the attack vector is modeled as $a_{k}^{u}=4$ from step $k=20$ to $k=35$, and so

$$
\tilde{u}_{k}=u_{k}+4 .
$$

From (37), it can be concluded that this attack is directly applied to the last two components of the state, i.e., $x_{3}$ and $x_{4}$. Therefore, the attack on the control signal can be detected by projecting the prediction ellipsoid set and the estimation ellipsoid set updated with the previous time instant onto the $x_{3}-x_{4}$ phase-plane.

Fig. 6 shows the sequence of the intersection between these two sets during the simulation time. As the bias injection attack starts at $k=20$, the center of the prediction ellipsoid set (6) is affected by the attack; however, the attack does not have any effect on the previous updated estimation ellipsoid set since it is based on the prediction set at step $k=19$. Therefore, it is expected from the subalgorithm 1a that there must be no intersection between the prediction ellipsoid set and the previous updated estimation ellipsoid set when the attack starts at $k=20$, i.e., $\chi_{21 \mid 20} \cap \chi_{20 \mid 20}=\varnothing$, as depicted in Fig. 7(b). 
From Remark 4, since there is no intersection between two sets from $k=20$ till $k=35$, then $\chi_{k+1 \mid k} \leftarrow \chi_{k \mid k}$ from $k=20$ to $k=35$ and as a result, the estimation ellipsoid set remains same as its updated set at $k=20$. Therefore, when the attack finishes at $k=36$, the center of the prediction ellipsoid set is based on the control signal at $k=36$ which is free of the attack, and the estimation ellipsoid set is same as its updated set at $k=20$ which is calculated from the prediction ellipsoid set obtained at $k=19$. Since the center of the prediction ellipsoid set at $k=19$ is not affected by the attack, from the subalgorithm $1 \mathrm{a}$, there exists intersection between these two sets at $k=36$, i.e., $\chi_{37 \mid 36} \cap \chi_{36 \mid 36} \neq \varnothing$, as shown in Fig. 7(c).

At $k=19$ the system is free of the attack, so from the subalgorithm 1a it can be concluded that there exists intersection between the two sets as illustrated in Fig. 7(a).

\section{CONCLUSION}

A novel cyber attack detection method based on the ellipsoidal set-membership filtering approach in NCSs is developed. Two ellipsoid sets are calculated for the system state in two steps: the prediction step and the measurement update step. The attack detection method relies on the intersection of the two sets resulting in two detection criteria. From subalgorithm 1a, the cyber attack on control signals can be detected if the prediction ellipsoid set does not have intersection with the estimation set updated with the measurement data received at the previous time instant. The subalgorithm $1 \mathrm{~b}$ indicates that the cyber attack on sensors measurements can be detected if there is no intersection between the prediction ellipsoid set and the estimation ellipsoid set updated with the measurement data obtained at the current time instant. A practical system is considered to show the effectiveness of the proposed attack detection method. In practical situations, when the system is subjected to the UBB noises, certain attack detectors such as the widely used $\chi^{2}$-detector cannot be effective. Therefore, it is of practical importance to provide a novel method that is able to detect cyber attacks on NCSs in which noises are not in the stochastic framework.

\section{REFERENCES}

[1] X. Yu and Y. Xue, "Smart grids: A cyber-physical systems perspective," Proc. IEEE, vol. 104, no. 5, pp. 1058-1070, May 2016.

[2] D. Wu, X.-M. Sun, Y. Tan, and W. Wang, "On designing eventtriggered schemes for networked control systems subject to one-step packet dropout," IEEE Trans. Ind. Informat., vol. 12, no. 3, pp. 902-910, Jun. 2016.

[3] X. Ge, F. Yang, and Q.-L. Han, "Distributed networked control systems: A brief overview," Inf. Sci., vol. 380, pp. 117-131, Feb. 2017.

[4] X.-M. Zhang, Q.-L. Han, and X. Yu, "Survey on recent advances in networked control systems," IEEE Trans. Ind. Informat., vol. 12, no. 5, pp. 1740-1752, Oct. 2016.

[5] R. A. Gupta and M.-Y. Chow, "Networked control system: Overview and research trends," IEEE Trans. Ind. Electron., vol. 57, no. 7, pp. 2527-2535, Jul. 2010.

[6] Y.-L. Wang and Q.-L. Han, "Network-based modelling and dynamic output feedback control for unmanned marine vehicles in network environments," Automatica, vol. 91, pp. 43-53, May 2018.

[7] Z. Zuo, Q.-L. Han, B. Ning, X. Ge, and X.-M. Zhang, "An overview of recent advances in fixed-time cooperative control of multi-agent systems," IEEE Trans. Ind. Informat., vol. 14, no. 6, pp. 2322-2334, Jun. 2018, doi: 10.1109/TII.2018.2817248
[8] L. Ma, Z. Wang, Q.-L. Han, and H.-K. Lam, "Variance-constrained distributed filtering for time-varying systems with multiplicative noises and deception attacks over sensor networks," IEEE Sensors J., vol. 17, no. 7, pp. 2279-2288, Apr. 2017.

[9] D. Ding, Q.-L. Han, Y. Xiang, X. Ge, and X.-M. Zhang, "A survey on security control and attack detection for industrial cyber-physical systems," Neurocomputing, vol. 275, pp. 1674-1683, Jan. 2018.

[10] L. Hu, Z. Wang, Q.-L. Han, and X. Liu, "State estimation under false data injection attacks: Security analysis and system protection," Automatica, vol. 87, pp. 176-183, Jan. 2018.

[11] D. Ding, Z. Wang, Q.-L. Han, and G. Wei, "Security control for discretetime stochastic nonlinear systems subject to deception attacks," IEEE Trans. Syst., Man, Cybern., Syst., vol. 48, no. 5, pp. 779-789, May 2018.

[12] J. Slay and M. Miller, "Lessons learned from the Maroochy water breach," in Critical Infrastructure Protection (IFIP International Federation for Information Processing), vol. 253, E. Goetz and S. Shenoi, Eds. Boston, MA, USA: Springer, 2008, pp. 73-82.

[13] S. Kuvshinkova, SQL Slammer Worm Lessons Learned for Consideration by the Electricity Sector, North Amer. Elect. Rel. Council, Atlanta, GA, USA, 2003.

[14] J. Farwell and R. Rohozinski, "Stuxnet and the future of cyber war," Survival, vol. 53, no. 1, pp. 23-40, 2011.

[15] A. A. Cárdenas, S. Amin, and S. Sastry, "Research challenges for the security of control systems," in Proc. 3rd Conf. Hot Topics Security, San Jose, CA, USA, Jul. 2008, pp. 1-6.

[16] S. T. Sarasamma and Q. A. Zhu, "Min-max hyperellipsoidal clustering for anomaly detection in network security," IEEE Trans. Syst., Man, Cybern. B, Cybern., vol. 36, no. 4, pp. 887-901, Aug. 2006.

[17] Z. Yu, J. J. P. Tsai, and T. Weigert, "An automatically tuning intrusion detection system," IEEE Trans. Syst., Man, Cybern. B, Cybern., vol. 37, no. 2, pp. 373-384, Apr. 2007.

[18] W. Hu, J. Gao, Y. Wang, O. Wu, and S. Maybank, "Online adaboostbased parameterized methods for dynamic distributed network intrusion detection," IEEE Trans. Cybern., vol. 44, no. 1, pp. 66-82, Jan. 2014.

[19] O. Y. Al-Jarrah et al., "Data randomization and cluster-based partitioning for botnet intrusion detection," IEEE Trans. Cybern., vol. 46, no. 8, pp. 1796-1806, Aug. 2016.

[20] Z.-H. Pang and G.-P. Liu, "Design and implementation of secure networked predictive control systems under deception attacks," IEEE Trans. Control Syst. Technol., vol. 20, no. 5, pp. 1334-1342, Sep. 2012.

[21] A. Rai, D. Ward, S. Roy, and S. Warnick, "Vulnerable links and secure architectures in the stabilization of networks of controlled dynamical systems," in Proc. Amer. Control Conf., Montreal, QC, Canada, Jun. 2012, pp. 1248-1253.

[22] A. S. Rawat, P. Anand, H. Chen, and P. K. Varshney, "Collaborative spectrum sensing in the presence of Byzantine attacks in cognitive radio networks," IEEE Trans. Signal Process., vol. 59, no. 2, pp. 774-786, Feb. 2011

[23] B. Kailkhura, Y. S. Han, S. Brahma, and P. K. Varshney, "Distributed Bayesian detection in the presence of Byzantine data," IEEE Trans. Signal Process., vol. 63, no. 19, pp. 5250-5263, Oct. 2015.

[24] O. Kosut, L. Jia, R. J. Thomas, and L. Tong, "Malicious data attacks on the smart grid," IEEE Trans. Smart Grid, vol. 2, no. 4, pp. 645-658, Dec. 2011.

[25] L. Liu, M. Esmalifalak, Q. Ding, V. A. Emesih, and Z. Han, "Detecting false data injection attacks on power grid by sparse optimization," IEEE Trans. Smart Grid, vol. 5, no. 2, pp. 612-621, Mar. 2014.

[26] G. Chaojun, P. Jirutitijaroen, and M. Motani, "Detecting false data injection attacks in AC state estimation," IEEE Trans. Smart Grid, vol. 6 , no. 5, pp. 2476-2483, Sep. 2015.

[27] B. M. Sanandaji, E. Bitar, K. Poolla, and T. L. Vincent, "An abrupt change detection heuristic with applications to cyber data attacks on power systems," in Proc. Amer. Control Conf., Portland, OR, USA, 2014, pp. 5056-5061.

[28] Y. Mo, R. Chabukswar, and B. Sinopoli, "Detecting integrity attacks on SCADA systems," IEEE Trans. Control Syst. Technol., vol. 22, no. 4, pp. 1396-1407, Jul. 2014.

[29] K. Manandhar, X. Cao, F. Hu, and Y. Liu, "Detection of faults and attacks including false data injection attack in smart grid using Kalman filter," IEEE Trans. Control Netw. Syst., vol. 1, no. 4, pp. 370-379, Dec. 2014.

[30] Y. Mo, S. Weerakkody, and B. Sinopoli, "Physical authentication of control systems: Designing watermarked control inputs to detect counterfeit sensor outputs," IEEE Control Syst., vol. 35, no. 1, pp. 93-109, Feb. 2015. 
[31] D. B. Rawat and C. Bajracharya, "Detection of false data injection attacks in smart grid communication systems," IEEE Signal Process. Lett., vol. 22, no. 10, pp. 1652-1656, Oct. 2015.

[32] Z. Feng, G. Wen, and G. Hu, "Distributed secure coordinated control for multiagent systems under strategic attacks," IEEE Trans. Cybern., vol. 47, no. 5, pp. 1273-1284, May 2017.

[33] A. B. Kurzhanski and I. Vályi, Ellipsoidal Calculus for Estimation and Control, 1st ed. Boston, MA, USA: Birkhäuser Basel, 1997.

[34] F. C. Schweppe, "Recursive state estimation: Unknown but bounded errors and system inputs," IEEE Trans. Autom. Control, vol. AC-13, no. 1, pp. 22-28, Feb. 1968.

[35] D. Bertsekas and I. Rhodes, "Recursive state estimation for a setmembership description of uncertainty," IEEE Trans. Autom. Control, vol. AC-16, no. 2, pp. 117-128, Apr. 1971.

[36] F. Yang and Y. Li, "Set-membership filtering for systems with sensor saturation," Automatica, vol. 45, no. 8, pp. 1896-1902, 2009.

[37] Z. Wu, F. Yang, and Q.-L. Han, "A novel islanding fault detection for distributed generation systems," Int. J. Robust Nonlin., vol. 24, nos. 8-9, pp. 1431-1445, 2014.

[38] X. Ge, Q.-L. Han, and F. Yang, "Event-based set-membership leaderfollowing consensus of networked multi-agent systems subject to limited communication resources and unknown-but-bounded noise," IEEE Trans. Ind. Electron., vol. 64, no. 6, pp. 5045-5054, Jun. 2017.

[39] N. Xia, F. Yang, and Q.-L. Han, "Distributed event-triggered networked set-membership filtering with partial information transmission," IET Control Theory Appl., vol. 11, no. 2, pp. 155-163, Jan. 2017.

[40] F. Yang, N. Xia, and Q.-L. Han, "Event-based networked islanding detection for distributed solar PV generation systems," IEEE Trans. Ind. Informat., vol. 13, no. 1, pp. 322-329, Feb. 2017.

[41] F. Yang and Y. Li, "Set-membership filtering with state constraints," IEEE Trans. Aerosp. Electron. Syst., vol. 45, no. 4, pp. 1619-1629, Oct. 2009.

[42] F. Yang and Y. Li, "Set-membership filtering for discrete-time systems with nonlinear equality constraints," IEEE Trans. Aerosp. Electron. Syst., vol. 54, no. 10, pp. 2480-2486, Oct. 2009.

[43] X. Ge, Q.-L. Han, and Z. Wang, "A dynamic event-triggered transmission scheme for distributed set-membership estimation over wireless sensor networks," IEEE Trans. Cybern., to be published, doi: 10.1109/TCYB.2017.2769722.

[44] L. El Ghaoui and G. Calafiore, "Robust filtering for discrete-time systems with bounded noise and parametric uncertainty," IEEE Trans. Autom. Control, vol. 46, no. 7, pp. 1084-1089, Jul. 2001.

[45] L. Ding, Q.-L. Han, X. Ge, and X.-M. Zhang, "An overview of recent advances in event-triggered consensus of multiagent systems," IEEE Trans. Cybern., vol. 48, no. 4, pp. 1110-1123, Apr. 2018.

[46] X. Ge, Q.-L. Han, D. Ding, X.-M. Zhang, and B. Ning, "A survey on recent advances in distributed sampled-data cooperative control of multiagent systems," Neurocomputing, vol. 275, pp. 1684-1701, Jan. 2018.

[47] R. E. Skelton, T. Iwasaki, and K. Grigoriadis, A Unified Algebraic Approach to Linear Control Design, Bristol, PA, USA: Taylor \& Francis, 1998.

[48] L. Vandenberghe and S. Boyd, "Semidefinite programming," SIAM Rev., vol. 38, no. 1, pp. 49-95, 1996.

[49] D. Simon and T. L. Chia, "Kalman filtering with state equality constraints," IEEE Trans. Aerosp. Electron. Syst., vol. 38, no. 1, pp. 128-136, Jan. 2002.

[50] A. Teixeira, D. Pérez, H. Sandberg, and K. H. Johansson, "Attack models and scenarios for networked control systems," in Proc. 1st Int. Conf. High Confidence Netw. Syst., Beijing, China, Apr. 2012, pp. 55-64.

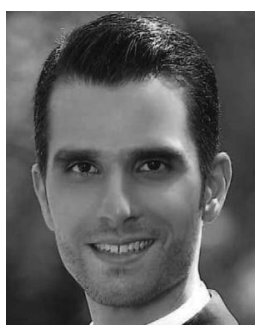

Eman Mousavinejad (S'14) received the B.Sc degree in mechanical engineering from Azad University, Tehran, Iran, in 2006, the M.Sc. degree in mechatronics engineering (control) from the Sharif University of Technology, Tehran, Iran, in 2009, and the M.Phil. degree in mechatronics engineering (control) from Griffith University, Gold Coast, QLD, Australia, in 2014, where he is currently pursuing the Doctor of Philosophy degree in mechatronics engineering (control) with the Griffith School of Engineering.

His current research interests include networked control and filtering, multiagent systems, cyber security, vehicle dynamics and control, autonomous vehicles, drive assistance systems, by-wire systems, and linear and nonlinear systems.

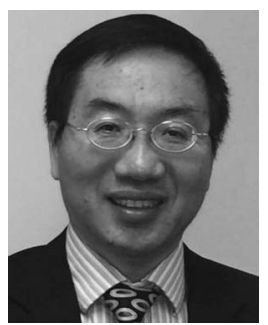

Fuwen Yang (M'99-SM'04) received the Ph.D. degree in control engineering from the Huazhong University of Science and Technology, Wuhan, China, in 1990

$\mathrm{He}$ is currently an Associate Professor with the Griffith School of Engineering, Griffith University, Gold Coast, QLD, Australia. He was a Research Fellow with Brunel University London, Uxbridge, U.K., and King's College London, London, U.K., a Professor with Fuzhou University, Fuzhou, China, and the East China University of Science and Technology, Shanghai, China, and an Associate Professor with Central Queensland University, Rockhampton, QLD, Australia. He had also held visiting professor appointments with the University of Manchester, Manchester, U.K., and the University of Hong Kong, Hong Kong. He has published over 200 journal and conference papers. His current research interests include networked control systems, distributed filtering and sensing, reliable fault detection and diagnosis, distributed control and filtering for smart girds, and solar PV power generation systems.

Dr. Yang is an Associate Editor of the IEEE CSS Conference Editorial Board.

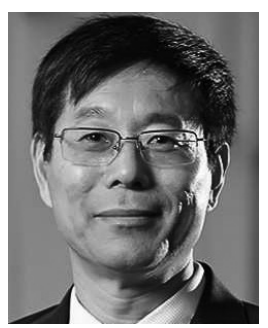

Qing-Long Han (M'09-SM'13) received the B.Sc. degree in mathematics from Shandong Normal University, Jinan, China, in 1983, and the M.Sc. and $\mathrm{Ph} . \mathrm{D}$. degrees in control engineering and electrical engineering from the East China University of Science and Technology, Shanghai, China, in 1992 and 1997, respectively.

From 1997 to 1998, he was a Post-Doctoral Researcher Fellow with the Laboratoire d'Automatique et d'Informatique Industielle (currently, Laboratoire d'Informatique et d'Automatique pour les Systémes), École Supérieure d'Ing'enieurs de Poitiers (currently, École Nationale Supérieure d'Ingénieurs de Poitiers), Université de Poitiers, Poitiers, France. From 1999 to 2001, he was a Research Assistant Professor with the Department of Mechanical and Industrial Engineering, Southern Illinois University at Edwardsville, Edwardsville, IL, USA. From 2001 to 2014, he was a Laureate Professor, an Associate Dean of Research and Innovation with the Higher Education Division, and the Founding Director of the Centre for Intelligent and Networked Systems, Central Queensland University, Rockhampton, QLD, Australia. From 2014 to 2016, he was the Deputy Dean of Research with the Griffith Sciences, and a Professor with the Griffith School of Engineering, Griffith University, Gold Coast, QLD, Australia. In 2016, he joined the Swinburne University of Technology, Melbourne, VIC, Australia, where he is currently the Pro Vice-Chancellor of Research Quality and a Distinguished Professor. In 2010, he was appointed as a Chang Jiang (Yangtze River) Scholar Chair Professor by the Ministry of Education, Beijing, China. His current research interests include networked control systems, time-delay systems, multi-agent systems, neural networks, and complex dynamical systems.

Prof. Han was a recipient of the one of the World's Most Influential Scientific Minds: 2014-2016, and the Highly Cited Researcher Award in Engineering by Thomson Reuters. He is an Associate Editor of a number of international journals, including the IEEE TRANSACTIONS ON INDUSTRIAL ELECTRONICS, the IEEE TRANSACTIONS ON INDUSTRIAL INFORMATICS, the IEEE TRANSACTIONS ON CYBERNETICS, and Information Sciences. He is a fellow of the Institution of Engineers Australia.

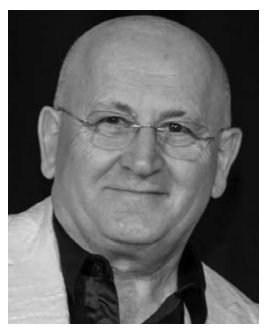

Ljubo Vlacic (M'92-SM'96) received the Graduate Diploma degree in electrical engineering and the M.Phil. and Ph.D. degrees in control systems engineering from the University of Sarajevo, Sarajevo, Bosnia and Herzegovina, in 1973, 1976, and 1986, respectively.

$\mathrm{He}$ is with the Institute for Integrated and Intelligent Systems, Griffith University, Gold Coast, QLD, Australia. He is a Control Systems Scientist and a Practitioner, renowned for his contributions to co-operative driverless vehicles and intelligent control systems research and development. He has held a number of leading roles in both industry and academia.

Prof. Vlacic was a recipient of 19 awards, including the IEE Achievement Medal (world-wide), the Sir Lionel Hooke Award, the Queensland Professional Engineer of the Year Award, and the Gold Coast Business Events Ambassador Award. His research achievements made news headlines and were broadcast through media outlets throughout the world. He is currently the Editor-in-Chief of the IEEE Intelligent Transportation Systems Magazine, the Chair of the Board, Engineers Australia ITEE College and the IET Network Queensland, and the General Chair of the 2019 IEEE Intelligent Transportation Systems Conference. He hosted ten national and international scientific conferences. 